\title{
Editorial
}

\section{Advanced modelling of human movements using numerical optimisation}

Ever since the pioneering studies carried out at the Physiology Station in the nineteenth century by Étienne-Jules Marey and Georges Demenÿ, the measurement devices and calculation techniques have evolved only so much for a long period. In order to achieve that, it was necessary to overcome the hurdles linked to the ability to record a great number of experimental data and treat them in an iterative fashion according to time and record frequency. Indeed, the mathematical models underlying the analysis of human dynamics (descriptive model) present a great deal of mechanical variables. The advancements in technology in the past 30 years - especially in the digital world - have enables us to gain access to these variables in an almost real time. At the same time, the simulation of human movements (predictive model) has developed in the wake of theoretical works that began in the 1940s in one of the sectors of mathematics called optimisation. Optimisation consists in determining the extreme of a function with or without a constraint. In other words, it means resolving an under-determined system, where the number of unknowns is superior to that of the equation. The simulation of a movement allows an answer to many research-related questions, such as the understanding of the mechanics of human movement, gesture technique optimisation and movement control. These studies demonstrate their relevance when they are complementary to experimental studies, as they explore a vast array of solutions. Numerical optimisation is also included in the descriptive models in order to enhance the entry variables of the dynamic model (identification of joint centres, joint kinematics reconstruction, etc.), or to enhance the estimation of force generated by the musculotendon complex. It goes without saying that these models rely on mathematics, ad it is of the utmost importance to fully understand them in order to correctly grasp their limits and implications.

The goal of this special issue is to provide a snapshot of the current use of numerical optimisation methods in predictive, as well as descriptive models. For it, we have asked researchers of international renown in the use of these mathematical methods applied to the biomechanics of the human movements. I would like to thank all the authors who have contributed to this special issue, as well as the whole body of experts that have duly and wonderfully acquitted themselves of this very delicate task. Through their observations and suggestions, they were able to enhance again the scientific quality of the contributions. This special issue made up of synthesis articles and original publications. All offer an innovating point of view on the use of these numerical methods of optimisation, and outline their future uses.

This issue is divided in two sections, the first one dealing with descriptive models of movement. It offers a relevant vision of the integration of numerical enhancement methods in order to access the muscular forces generated during movement or joint kinematics. A final contribution focuses on the relevance of the entry data of these models.

- Laurence Chèze, Florent Moissenet and Raphaël Dumas (Université de Lyon, France and Rehazenter, Luxembourg) offer a synthesis of the main methods of prediction in musculo-tendon forces currently available in the scientific literature. These methods are described and their limits pointed out. What emerges from their synthesis is that further development is necessary before applying the musculo-tendon forces predicted by this type of models for clinical purposes.

- Vincent Fohanno, Patrick Lacouture and Floren Colloud (Université de Poitiers and Université de Nantes, France) are interested in the reconstruction of joint kinematics based on a global optimisation technique. They show that the number of markers placed on the subject leads to a modification of the associated joint kinematics. They conclude that the kinematic variables must be considered cautiously when the experimental design consists of a limited number of markers. 
- Franck Quaine, Florent Paclet, Laurent Vigouroux and François Moutet (Université de Grenoble, Aix-Marseille Université, France and The Pennsylvania State University, USA) question the integration of theories issued from motor control in their calculation process of muscular forces generated by the extrinsic muscles of the hand during complex tasks in order to better understand the experimentally observed muscular co-contractions. Thus, the principle of "secondary moment minimisation" - in this case, the balance degrees of freedom of the wrist joint is added to the equation set of their model. Three different experimental conditions are put into practice (intact hand, paralysed hand with radial nerve palsy and a transferred hand after tendon transfer (the so-called Tsuge technique)) in order to modify the muscular capacity while at the same time maintaining identical secondary moments. Their results underline the fact that the integration of this minimising principle of secondary moments enhances the biomechanical models in their ability to show and explain co-contractions.

- Mariana Kersh, Heidi-Lynn Ploeg and Marcus Pandy (University of Melbourne, Australia and University of Wisconsin, USA) present a synthesis of the works on the contribution of cruciate ligaments in knee stability. Relying on experimental data and mathematical modelling, they quantify the role of these different structures in knee stability, depending on the joint flexion angle. These data are of utmost importance in the improvement of predictive and descriptive models of the knee, the development of an effective protocol after an injury and the conception of a prosthetic knee.

The second section underlines every interest in the numerical simulation of the human movement. These models have many simplifications of the system they imitate, but they are as many powerful tools, allowing to test various hypotheses and prove the mechanisms that underlie the complex coordination of the musculoskeletal system. From this point of view, they are highly complementary of the descriptive models.

- Mark King and Maurice Yeadon (Loughborough University, UK) present the theoretical foundations of predictive models developed in their research facility over the past 20 years. Sports movement simulation such as aerial movement, high bar, tumbling, running jumps for heigh and distance, spring board diving takeoffs, drop landings, vaulting or vertical jumping provide insight into the mechanics behind sport movements. They provide the various components for each model, the subject-specific parameters, model evaluation, the key findings and the strengths / limitations. Finally, they put into perspective how models could further progress in the future.
- John Challis and Zachary Domire (The Pennsylvania State University and East Carolina University, USA) show in this three-study review the interest in numerical simulation in order to examine the coordination of the musculoskeletal system. In the course of these three studies, the maximum vertical jumping performance is simulated using a direct dynamics muscle driven model. The first study highlights how their model can be used to guide technique modifications in order to optimise performance. The second study shows how muscular properties impose jumping performance. Finally, the third study explains how the armswing influences this performance.

- Frédéric Marin (Université de Technologie de Compiègne, France) proposes a musculoskeletal predictive modelling of the cervical spine. A mechanical connector combined with a visco-elastic component classically represents the functional spinal units. The author takes particular interest in the influence these two parameters have on the results of the movement simulation of the cervical spine, and shows the great sensitivity of the model to the two parameters. There, up to this day, the results of these simulations must be considered with extreme caution.

- Arnaud Hamon and Yannick Aoustin (École Centrale de Nantes, France) are interested in the design of a knee-joint in a bipedal robot drawing inspiration on the degrees of freedom observed in the human knee. They offer an innovating concept of this joint based on a four-bar linkage allowing a translation of the instantaneous centre of rotation. A cyclic, stable walking gait of the robot in a sagittal plane is generated by an parametric optimisation associated with a sthenic criterion. The use of this new joint decreases the knee joint torque when compared to a classic revolution joint. It notably influences the distribution of energy between simple and double support phases, thus growing even closer to the characteristics of human walking.

- Chris Hayot, Sophie Sakka, Vincent Fohanno and Patrick Lacouture (Université de Poitiers and École Centrale de Nantes, France) are interested in the simulation of the centre of mass trajectory during walking, using a three-dimensional Extended Inverted Pendulum model. In order to improve the accuracy of their output variables, they consider an original contact model allowing the displacement of the pivot point during the single support phase, as well as during the double support phase. This new model accurately predicts the lateral displacements of the centre of mass, regardless of the combination of walking speed/stride length/stride width chosen by the subject.

All these contributions show the wealth these mathematical methods have to offer and how they stimulate the 
development of more and more realistic models in the field of biomechanics. Once they validated, these models become, in a very short lapse of time, transposable to broader, more general questions including approaches to motor control, cognition, physiology of movement, etc. They can only improve our knowledge of complex systems that underlie the generation of a coherent and coordinated movement, involving several segments.

I trust you will all enjoy reading the nine contributions making up this special issue.

Floren Colloud Guest Editor 


\section{Éditorial}

\section{Modélisation du mouvement humain par des méthodes numériques d'optimisation}

Depuis les travaux précurseurs menés à la station de physiologie à la fin du $19^{\text {e }}$ siècle par Étienne-Jules Marey et Georges Demenÿ, les outils de mesure et les techniques calculatoires n'ont finalement que peu évolué pendant une longue période. Pour cela, il était nécessaire de lever plusieurs verrous essentiellement liés à la capacité à enregistrer un grand nombre de données expérimentales et à traiter ces données de façon itérative en fonction du temps et de la fréquence d'enregistrement. En effet, les modèles mathématiques sous-jacents à l'analyse de la dynamique du mouvement humain (modèle descriptif) comportent un nombre important de variables mécaniques. Les avancées technologiques de ces 30 dernières années, notamment dans le domaine du numérique, nous permettent actuellement d'accéder à ces variables selon un flux quasi temps réel. Parallèlement, la simulation des mouvements humain (modèle prédictif) s'est développée suite aux travaux théoriques qui ont débuté au cours des années 1940 dans une branche des mathématiques appelée optimisation. L'optimisation consiste à déterminer l'extremum d'une fonction avec ou sans contrainte. En d'autres termes, il s'agit de résoudre un système sous-déterminé, le nombre d'inconnu étant supérieur au nombre d'équation. La simulation du mouvement permet de répondre à de nombreuses questions de recherche telles que la compréhension de la mécanique des mouvements humains, l'optimisation de la technique gestuelle et le contrôle du mouvement. Ces études révèlent toute leur pertinence lorsqu'elles sont complémentaires d'études expérimentales car elles permettent d'explorer un grand nombre de solutions. L'optimisation numérique est aussi intégrée dans des modèles descriptif afin d'améliorer les variables d'entrée du modèle dynamique (détermination des centres articulaires, reconstruction de la cinématique articulaire, etc.) ou l'estimation des forces générées par le complexe musculo-tendineux. Il n'en reste pas moins que ces modèles reposent sur des mathématiques qu'il est important de bien comprendre afin d'appréhender correctement leur limites et leurs implications.

L'objectif de ce numéro spécial est de fournir une photographie de l'utilisation actuelle des méthodes numériques d'optimisation tant pour des modèles prédictifs que pour des modèles descriptifs. Nous avons pour cela sollicité des chercheurs reconnus internationalement dans l'utilisation de ces méthodes mathématiques en biomécanique du mouvement humain. Je tiens à remercier l'ensemble des auteurs qui ont contribué à ce numéro spécial. Je voudrais également remercier l'ensemble des experts qui se sont acquittés avec sérieux et brio de cette tâche très délicate. Ils ont su, par leurs remarques et suggestions, améliorer encore la qualité scientifique de ces contributions. Ce numéro spécial est composé d'articles de synthèse et d'articles originaux. Tous apportent un point de vue novateur sur l'utilisation de ces méthodes numériques d'optimisation et dressent des esquisses de leurs utilisation dans le futur.

Ce numéro spécial est structuré en deux sections. La première section porte sur les modèles descriptifs du mouvement. Elle offre une vision pertinente de l'intégration des méthodes d'optimisation numérique pour accéder aux forces musculaires générées au cours du mouvement ou à la cinématique articulaire. Une dernière contribution porte l'accent sur la pertinence des données d'entrée de ces modèles.

- Laurence Chèze, Florent Moissenet et Raphaël Dumas (Université de Lyon, France et Rehazenter, Luxembourg) proposent une synthèse des principales méthodes de prédiction des forces musculotendineuses actuellement disponibles dans la littérature scientifique. Ces méthodes sont décrites et leurs limites soulignées. Ils ressort de cette synthèse que de nouveaux développements sont encore nécessaires avant que les forces musculo-tendineuses prédites par ces modèles puissent être utilisées pour des applications en clinique.

- Vincent Fohanno, Patrick Lacouture et Floren Colloud (Université de Poitiers et Université de Nantes, France) s'intéressent à la reconstruction de la cinématique articulaire basée sur une technique d'optimisation globale. Ils montrent que le nombre de marqueurs placés sur le sujet a pour conséquence une modification de la cinématique articulaire associée. 
Ils concluent que les variables cinématiques doivent être analysées avec prudence lorsque le dispositif expérimental comprend un faible nombre de marqueurs.

- Mariana Kersh, Heidi-Lynn Ploeg et Marcus Pandy (University of Melbourne, Australia et University of Wisconsin, USA) présentent une synthèse des travaux portant sur la contribution des ligaments croisés et latéraux à la stabilité du genou. S'appuyant sur des données expérimentales et des modélisations mathématiques, ils quantifient le rôle de ces différentes structures dans la stabilité du genou en fonction de l'angle de flexion. Ces données sont de premières importances pour améliorer les modèles prédictifs et descriptifs du genou, la mise en place de protocole efficace suite à une lésion et la conception de prothèse du genou.

- Franck Quaine, Florent Paclet, Laurent Vigouroux et François Moutet (Université de Grenoble, Aix-Marseille Université, France et The Pennsylvania State University, USA) questionnent l'intégration de théories issues du contrôle moteur dans leur processus calculatoire des forces musculaires générées par les muscles extrinsèques de la main lors de tâches complexes afin de mieux appréhender les co-contractions musculaires observées expérimentalement. Ainsi, le principe de «minimisation des moments secondaires », dans ce cas l'équilibre des degrés de liberté de l'articulation du poignet, est ajouté dans le set d'équations de leur modèle. Trois conditions expérimentales différentes (main intacte, main paralysée suite à une atteinte du nerf radial et main réanimée suite à un transfert tendineux (technique dite de «Tsugé »)) sont mises en place afin de modifier les capacités musculaires tout en gardant des moments secondaires identiques. Leur résultats mettent en évidence que l'intégration de ce principe de minimisation des moments secondaires améliore les modèles biomécaniques dans leur capacité à montrer et expliquer les co-contractions.

La seconde section montre tout l'intérêt de la simulation numérique du mouvement humain. Ces modèles comportent de nombreuses simplifications du système qu'ils imitent, il n'en reste pas moins des outils puissants permettant de tester de nombreuses hypothèses et de démontrer les mécanismes qui sous-tendent la coordination complexe du système musculo-squelettique. Ils sont en cela extrêmement complémentaires des modèles descriptifs.

- Mark King et Maurice Yeadon (Loughborough University, UK) présentent les fondements théoriques des modèles prédictifs mis au point au cours de ces 20 dernières années dans leur groupe de recherche. La simulation de mouvements sportifs tels que les mouvements aériens, la barre fixe, le tumbling, la réception de sauts, l'impulsion en plongeon, le cheval d'arçon, le triple sauts ou encore l'impulsion d'un saut lors d'une course illustrent leurs propos. Pour chaque modèle, ils précisent leurs différents éléments, la personnalisation des paramètres, l'évaluation du modèle, les résultats clés et les points forts/limites. Enfin, ils mettent en perspective la façon dont ces modèles pourraient évoluer dans le futur.

- John Challis et Zachary Domire (The Pennsylvania State University et East Carolina University, USA) montrent dans cette synthèse de trois études l'intérêt de la simulation numérique pour caractériser la coordination du système musculo-squelettique. Lors de ces trois études, la performance maximale en saut vertical est simulée à l'aide d'un modèle dynamique direct planaire actionnée par des muscles. La première étude met en évidence comment leur modèle peut être utilisé pour élaborer des modifications techniques afin d'optimiser la performance. La seconde étude illustre comment les propriétés musculaires dictent la performance du saut. Enfin, la troisième étude explique comment le balancement des membres supérieurs influence cette performance.

- Frédéric Marin (Université de Technologie de Compiègne, France) propose un modèle musculosquelettique prédictif du rachis cervical. L'unité fonctionnelle du rachis cervical est classiquement modélisée par une liaison mécanique comprenant une composante visco-élastique. L'auteur s'intéresse plus particulièrement à l'influence de ces deux paramètres sur les résultats issus de simulations du mouvement du rachis cervical. Il montre la grande sensibilité de ce modèle à ces deux paramètres. Ainsi, à ce jour, les résultats de ces simulations doivent être interprétés avec les plus grandes précautions.

- Arnaud Hamon et Yannick Aoustin (École Centrale de Nantes, France) s'intéressent à la conception de l'articulation du genou d'un robot bipède s'inspirant des degrés de liberté observés chez l'homme. Ils proposent une conception innovante de cette articulation basée sur une structure parallèle 4-barres autorisant le glissement du centre de rotation instantanée. Un mouvement de marche cyclique du robot dans le plan sagittal est généré par une optimisation paramétrique associée à un critère d'optimisation sthénique. L'implémentation de cette nouvelle articulation diminue le couple articulaire au genou en comparaison avec une articulation classique. Elle influence particulièrement la distribution d'énergie entre les phases de simple appui et les phases de double appuis se rapprochant ainsi des caractéristiques de la marche humaine.

- Chris Hayot, Sophie Sakka, Vincent Fohanno et Patrick Lacouture (Université de Poitiers et École Centrale de Nantes, France) s'intéressent à la simulation de la trajectoire du centre de masse au cours de la marche par un modèle pendule inversé tridimensionnel. Afin d'améliorer la finesse des résultats, ils proposent un modèle de contact original autorisant le 
déplacement du point de pivot aussi bien lors de la phase de simple appui que lors de la phase de double d'appui. Ce nouveau modèle prédit remarquablement les déplacements latéraux du centre de masse quelle que soit la combinaison vitesse de marche/longueur de pas/largeur de pas choisie par le sujet.

L'ensemble de ces contributions montre la richesse qu'offre ces méthodes mathématiques et comment elles stimulent le développement de modèle de plus en plus réalistes dans le champ de la biomécanique. Une fois validés, ces modèles peuvent être transposables dans un laps de temps très court à des questionnements plus généraux comprenant des approches en contrôle moteur, cognition, physiologie du mouvement, etc. Ils contribuent à améliorer notre niveau de connaissances des systèmes complexes qui sous-tendent la génération d'un mouvement cohérent et coordonné impliquant plusieurs segments.

Je vous souhaite une bonne lecture des neuf contributions constituant ce numéro spécial.

Floren Colloud Éditeur invité 